\title{
Invariant Manifolds and Cluster Synchronization in a Family of Locally Coupled Map Lattices
}

\author{
VLADIMIR BELYKH ${ }^{\mathrm{a}, *}$, IGOR BELYKH $^{\mathrm{b}}$, NIKOLAI KOMRAKOV $^{\mathrm{a}}$ and ERIK MOSEKILDE ${ }^{\mathrm{c}}$ \\ ${ }^{a}$ Department of Mathematics, Volga State Academy, 5, Nesterov Street, 603600 Nizhny Novgorod, Russia; \\ ${ }^{\mathrm{b}}$ Department of Differential Equations, Institute of Applied Mathematics, 10, Ul'yanov Street, 603005 Nizhny Novgorod, \\ Russia; ${ }^{\mathrm{c}}$ Department of Physics, Technical University of Denmark, 2800 Lyngby, Denmark
}

\begin{abstract}
This paper presents an analysis of the invariant manifolds for a general family of locally coupled map lattices. These manifolds define the different types of full, partial, and antiphase chaotic synchronization that can arise in discrete dynamical systems. Existence of various invariant manifolds, self-similarity as well as orderings and embeddings of the manifolds of a coupled map array are established. A general variational equation for the stability analysis of invariant manifolds is derived, and stability conditions for full and partial chaotic synchronization of concrete coupled maps are obtained. The general results are illustrated through examples of three coupled two-dimensional standard maps with damping.
\end{abstract}

Keywords: Coupled chaotic maps, Invariant manifolds, Synchronization, Chaos, Embedding

\section{INTRODUCTION}

During the last $10-15$ years, coupled maps and coupled map lattices (CMLs) have come to play an essential role in the theory of dynamical systems as objects for which different properties of complex temporal and spatio-temporal behavior can be studied [1-11]. Among the most interesting phenomena that can arise in coupled map systems, full and partial synchronization, on-off intermittency, attractor bubbling, and riddled basins of attraction have been studied in numerous papers for different dynamical systems and from different points of view $[1,5-7,9-11]$. These phenomena are directly related to the existence of stable linear invariant manifolds to which the trajectories of the synchronous modes of groups (or clusters) of CML elements are restrained.

Our objective in the present paper is to study cluster synchronization and invariant manifolds for the general family of coupled identical and regularly arranged maps. A simple example of such a CML is the one-dimensional array of one-dimensional maps

$$
\bar{x}_{i}=f\left(x_{i}\right)+\varepsilon\left[g\left(x_{i-1}\right)-2 g\left(x_{i}\right)+g\left(x_{i+1}\right)\right],
$$

where $i=1,2, \ldots, N$ represents the discrete space coordinate, and a bar over the variable $x$ denotes

* Corresponding author. 
next iteration. $f: R^{1} \rightarrow R^{1}$ is the nonlinear map associated with the individual element, and $g: R^{1} \rightarrow R^{1}$ is a coupling function, with $\varepsilon$ being a measure of the strength of the coupling. For $g(x) \equiv x$, the system (1) reduces to the much studied diffusively CMLs [3,5,9], and for $g(x) \equiv f(x)$ we recover the so-called nonlinearly CMLs as studied, for instance, by Kaneko [1].

We have recently described a family of stable invariant manifolds for diffusively coupled systems of differential equations [13]. An example of such a system is a regular chain of coupled Rossler oscillators. The invariant manifolds serve as a frame for the possible dynamical behaviors of the coupled systems in phase space, defining in particular the various types of synchronized behavior that can occur. In the present paper we consider the existence and stability of the invariant manifolds that define the various states of partial synchronization (or cluster dynamics) for an array of elements of general multi-dimensional maps with local coupling.

Given the multi-dimensional single map $F: R^{m} \rightarrow R^{m}$

$$
\bar{x}=F(x), \quad x \in R^{m},
$$

we consider the $N$-dimensional array of identical elements having identical dynamics defined by the single map $F$ and with interaction with the neighboring elements through the coupling function $G$, i.e., we consider the $N \times m$-dimensional map $T$ :

$$
\bar{x}_{i}=F\left(x_{i}\right)+\varepsilon \cdot G\left(x_{i-1}, x_{i}, x_{i+1}\right),
$$

where $i=1,2, \ldots, N$. As before, the bar denotes next iterate (now of the vector variable $x$ ), and $\varepsilon$ is a measure of the coupling strength. The function $G(u, v, w)$ is assumed to be symmetric and to satisfy the conditions

$$
G(u, u, u)=0, \quad G(u, v, w)=G(w, v, u) .
$$

Similarly, to the function $g(x)$ in the simple array of one-dimensional maps (1), the coupling function $g$ allows for both a linear diffusive coupling with

$$
G=E_{p}\left(x_{i-1}-2 x_{i}+x_{i+1}\right),
$$

and for a nonlinear Kaneko-type coupling with

$$
G=E_{p}\left(F\left(x_{i-1}\right)-2 F\left(x_{i}\right)+F\left(x_{i+1}\right)\right) .
$$

In the form (2), the CML is locally coupled due to the assumed combination of three neighboring vector variables in the interaction term. However, as $G$ may be an arbitrary function satisfying a set of symmetry conditions similar to (3), our analysis may be extended to include long-range coupling. In this case the matrix $E_{p}$, which serves to pick up the combination of coupled vector variables, will have nonzero elements far from the main diagonal.

As previously noted, our aim is to study global aspects of the dynamics of the map $T$ related to the existence and stability of invariant manifolds defining the possible cluster synchronizations of the individual elements. Throughout the study we shall assume zero-flux boundary conditions, i.e.,

$$
x_{0} \equiv x_{1} \quad \text { and } \quad x_{N} \equiv x_{N+1} .
$$

The boundary conditions obviously play an essential role with respect to which manifolds can exist and be stable.

\section{MAIN INVARIANT MANIFOLDS}

Let us start by introducing the definition of an $n$-dimensional invariant manifold $M$ of an arbitrary $k$-dimensional map $\varphi$ :

$$
\bar{x}=\varphi(x), \quad x \in R^{k}, \varphi: R^{k} \rightarrow R^{k} .
$$

First we note that any $l$-dimensional manifold in $R^{k}$ is defined by the map

$$
x=C(u), \quad C: R^{l} \rightarrow R^{k},
$$

where $u \in R^{l}$ is taken as the vector of coordinates in the manifold, and $x=C(u)$ is a graph determining the $l$-dimensional manifold in $R^{k}$.

DEFINITION 1 The l-dimensional manifold $M$ is an invariant manifold of the $k$-dimensional map $\bar{x}=\varphi(x)$ if for any $x \in M$ the image $\bar{x}=\varphi(x) \in M$. The map $\left.\varphi\right|_{M}$ may be written in the form of the 
equation $C(\bar{u})=\varphi(C(u)), u \in R^{l}$ which must have a solution with respect to $\bar{u}$ being a map $\bar{u}=\tilde{\varphi}(u)$ acting in $M, \tilde{\varphi}: M \rightarrow M$.

Let now $I$ be an $m \times m$ unit matrix

$$
I=\left(\begin{array}{lll}
1 & & 0 \\
& \ddots & \\
0 & & 1
\end{array}\right)
$$

and let $C$ be an $N \times n$ block matrix such that each element of $C$ is either the $m \times m$ zero matrix or the unit matrix $I$, and each row of $C$ contains only one matrix $I$. Let $X=\left(x_{1}, x_{2}, \ldots, x_{N}\right)^{\mathrm{T}}$ denote a column vector, then the system (2) may formally be written as a multi-dimensional map

$$
\bar{X}=T(X) .
$$

From the general Definition 1 we obtain the definition of a linear manifold of the map (5).

DEFINITION 2 If there exists an integer $n \in[1, N]$, a column vector $U=\left(u_{1}, u_{2}, \ldots, u_{n}\right)^{\mathrm{T}}$, where $u_{j} \in R^{m}$, $j=1,2, \ldots, n$, and a correspondence matrix $C$ such that the map under $T$ under the linear transformation

$$
X=C U
$$

attains the form of $n$ compatible equations $C \bar{u}=T(C u)$, i.e., only $n$ out of the $N$ equations in (6) are linearly independent, then the map $T$ has an $n \times m$-dimensional invariant manifold $M(N, n)$ with the coordinates $\left(u_{1}, u_{2}, \ldots, u_{n}\right)$ in it.

The manifold $M(N, n)$ defines $n$ synchronous clusters of the array, such that the nonzero elements of the matrix $C$ pick out those elements from the array that form the $n$ clusters and define the size of each cluster.

As an example, we immediately introduce two well-known clusters:

(1) For $n=N, U=X, C$ is the unit matrix, and we obtain the manifold $M(N, N)$ being the phase space of the map $T$. Hence, the system (2) has $N$ independent elements ( $N$ clusters).
(2) For $n=1, U=x_{1}$ we obtain the "diagonal" manifold $M(N, 1)$ with the single map (1) in it due to the symmetry (3). In this case we have one cluster spatial dynamics exhibiting full synchronization of the array when the spatial sites are "all doing the same thing at the same moment, even though it is chaotic motion" [7].

The manifold $M(N, n)$ for $1<n<N$ corresponds to cluster synchronization when all elements of each of $n$ clusters are identical in their dynamics.

Let us hereafter study the problem of the existence of invariant manifolds for the map $T$.

It turns out that for the system (2) we can relate the first $n$ coordinates of vector $X$ to the variables in the manifold $M(N, n)$. Thus, we put $u_{j}=x_{j}$, $j=1,2, \ldots, n$, in the vector $U$.

Denote the unit $n \times n$ block matrices as

$$
E=\left(\begin{array}{lll}
I & & 0 \\
& \ddots & \\
0 & & I
\end{array}\right), \quad \bar{E}=\left(\begin{array}{lll}
0 & & I \\
I & & \\
I & & 0
\end{array}\right)
$$

and let int $(\zeta)$ denote the integer part of $\zeta$.

THEOREM 1 The map $T$ has an invariant manifold $M^{c}(N, n)$ with $n=\operatorname{int}((N+1) / 2)$,

$$
\begin{aligned}
& M^{c}(N, n)=\left\{x_{i}=x_{N-i+1},\right. \\
&i=1,2, \ldots, \operatorname{int}((N+1) / 2)\} .
\end{aligned}
$$

The correspondence relation (6) has the form

$$
X=C U=\left(\begin{array}{c}
E \\
\bar{E}
\end{array}\right) \cdot U
$$

for even $N=2 n$ and the form

$$
X=C U=\left(\begin{array}{cc}
E & 0 \\
0 & I \\
\bar{E} & 0
\end{array}\right) \cdot U
$$

for odd $N=2 n-1$.

The dynamical system in the invariant manifold $M^{c}(N, n)$ is defined by the system (2) where $N$ stands for int $((N+1) / 2)$ with zero-flux boundary conditions (4) for even $N$, and with nonsymmetrical boundary 
conditions

$$
x_{0}=x_{1}, \quad x_{n+1}=x_{n-1}
$$

for odd $N=2 n-1$.

Proof The proof of the theorem is straightforward by substitution of $X=C U$ into (2) with the condition (3).

The manifold $M^{c}(N, n)$ has the central symmetry with respect to the middle of the array and corresponds to the clustering in pairs (one central element for odd $N$ is a separate cluster).

THEOREM 2 Let $N=r \cdot n$, where $r$ and $n$ are arbitrary integers. Then the map $T$ has an invariant manifold

$$
\begin{aligned}
& M^{a}(N, n)=\left\{x_{i+2 n j}=x_{-i+1+2 n j}=x_{i},\right. \\
&i=1,2, \ldots, n, j=1,2, \ldots, r\} .
\end{aligned}
$$

The correspondence matrix $C$ has the form

$$
C=\underbrace{(E, \bar{E}, E, \ldots, \bar{E}, E, \bar{E}, \ldots)^{\mathrm{T}}}_{r \text { times }} .
$$

The dynamical system in the invariant manifold $M^{a}(N, n)$ is defined by the system (2), where $N$ stands for $n$ with zero-flux boundary conditions (4).

Proof The proof of the theorem is straightforward as well.

Corollary 1 For even $N=2 n$ the embedded manifold $M^{a}(N, n)=M(n, n)$, i.e., the manifold $M^{a}(N, n)$ may be considered as the phase space of the original system (2) with half the original dimension. Hence, Theorem 1 applies to $\left.T\right|_{M(N, n)}$ for even $N$.

Corollary 2 For odd $N=2 n-1$ the system (2) is not self-similar in the manifold $M^{a}(N, n)$ because of asymmetric boundary conditions (7) and Theorem 1 does not apply to $\left.T\right|_{M(N, n)}$. Hence, for $N=$ $p \cdot 2^{k}$, where $p$ is an odd number, we obtain the following ordering of dimensions of the embedded invariant manifolds:

$$
\begin{aligned}
p \cdot 2^{k} & \rightarrow p \cdot 2^{k-1} \rightarrow \cdots \rightarrow p \cdot 2 \\
& \rightarrow p \rightarrow \frac{p+1}{2} .
\end{aligned}
$$

The manifold $M^{a}(N, n)$ has an alternating symmetry, and each of the $n$ clusters has $r$ elements such that for even $r$ the first and the last elements of the array, $x_{1}$ and $x_{N}$, belong to the same cluster and $M^{a}(N, n)$ has the central symmetry with respect to the middle of array. Hence, for even $r$ the manifold $M^{a}(N, n)$ is related to the manifold $M^{c}(N, n)$ of Theorem 1. In this case, the recurrent rule of Theorem 1 is applicable to $\left.T\right|_{M^{a}(N, n)}$.

Remark For any composed number $N$, each cofactor of $N$ may stand for $n$ and defines the number of clusters. For example, the number,

$$
N=12=6 \cdot 2=4 \cdot 3=3 \cdot 4,
$$

and according to Theorem 2 we immediately obtain three different manifolds $M^{a}(12,2)$, $M^{a}(12,3)$ and $M^{a}(12,4)$.

\section{FAMILIES OF EMBEDDED INVARIANT MANIFOLDS}

From Theorems 1 and 2 it follows that independently from the individual dynamics defined by the single map $\bar{x}=F(x)$, the system of coupled maps (2), i.e. the map $T$, has invariant manifolds with the central symmetry $M^{c}$ as well as manifolds with the alternative symmetry $M^{a}$ which exist due to the symmetry of the coupling (3) and the symmetry of the boundary conditions (4). The significant feature of Theorems 1 and 2 is the recurrence due to self-similarity of invariant manifold dynamics. Together with the permutation of cofactors of $N$ in Theorem 2 this allows us to use these theorems as generating rules for obtaining families of invariant manifolds and studying their hierarchy. In this way we can determine the different spatial-temporal dynamical regimes of the complicated cooperative behavior of coupled maps.

THEOREM 3 Let $N=r \cdot n$, where $r$ and $n$ are arbitrary integers greater than 1 . Then the following 
enclosures are valid:

$$
\begin{aligned}
& M(N, N) \supset M^{a}(N, n) \\
& M^{c}\left(N, \operatorname{int}\left(\frac{N+1}{2}\right)\right) \supset M^{c}\left(N, \operatorname{int}\left(\frac{n+1}{2}\right)\right) .
\end{aligned}
$$

Proof For even $r, M^{a}(N, n)$ has the central symmetry and lies in $M^{c}(N, \operatorname{int}((N+1) / 2))\left(M^{a}\right.$ coincides with $M^{c}$ for $\left.r=2\right)$. The enclosure $M^{c}(N, \operatorname{int}((N+1) / 2)) \subset M^{a}(N, n)$ follows from Theorem 1 applied for the system in $M^{a}(N, n)$ being the system (2) where $N$ stands for $n$ (due to self-similarity). The enclosure

$$
M^{a}\left(N, \operatorname{int}\left(\frac{n+1}{2}\right)\right) \supset M^{a}\left(N, \operatorname{int}\left(\frac{N+1}{2}\right)\right)
$$

is valid because both manifolds have the central symmetry with respect to the middle of the array and because the upper part of matrix $C$ is the unit matrix $E$ so that the first $\operatorname{int}((N+1) / 2)$ sites of the array belong to both manifolds.

COROLlary If the maps $\left.T\right|_{M^{a}(N, n)}$ and $\backslash$ or $\left.T\right|_{M^{c}(N, \operatorname{int}((n+1) / 2))}$ satisfy Theorems 1 and 2 with

$$
\begin{aligned}
& M^{a}(N, n)=M(n, n), \\
M^{a}\left(N, \operatorname{int}\left(\frac{n+1}{2}\right)\right) & \\
= & M\left(\operatorname{int}\left(\frac{n+1}{2}\right), \operatorname{int}\left(\frac{n+1}{2}\right)\right),
\end{aligned}
$$

then the enclosures (15) are extendable.

Now we list the rules of embeddings based on Theorems 1-3.

Rule 1 If $N$ is a prime number $(N=2,3,5,7, \ldots)$ then there exists an invariant manifold

$$
M^{c}\left(N, \operatorname{int}\left(\frac{N+1}{2}\right)\right) \subset M(N, N)
$$

such that $\left.T\right|_{M^{c}}$ has asymmetrical boundary conditions (7) and Theorems 1-3 give only one next embedded manifold $M(N, 1)$ corresponding to synchronization of all individual elements of the array (2).

For example, if $N=7$, then the dimensions of embeddings follows as $7 \rightarrow 4 \rightarrow 1$.

Rule 2 Let $N=p^{k}$ where $p \geq 3$ is a prime number (for $p=2$, see (8)). Then we obtain an ordering

$$
\begin{gathered}
M\left(N, p^{k}\right) \supset M^{a}\left(\cdot, p^{k-1}\right) \supset M^{a}\left(\cdot, p^{k-2}\right) \supset \cdots \\
\supset M^{a}(\cdot, p) \supset M^{c}\left(\cdot, \frac{p+1}{2}\right) \supset M(N, 1)
\end{gathered}
$$

which follows from Theorem 2 with the last step from Rule 1. Due to Theorem 3, in accordance with (9), the ordering (10) is composed as follows:

$$
\begin{aligned}
\cdots & \underset{\cup}{M\left(N, p^{k-i}\right) \supset M\left(p^{k-i}, p^{k-i-1}\right)} \supset \cdots \\
& \supset M\left(p^{k-i}, \frac{p^{k-i}+1}{2}\right) \supset M\left(p^{k-i}, \frac{p^{k-i-1}+1}{2}\right), \\
& i=0,1, \ldots, k-1 .
\end{aligned}
$$

Rule 3 Let $N=q \cdot p^{k}$, where $p$ is a prime number and $q$ is an arbitrary integer. Then similarly to Rule 2 we have an ordering

$$
\begin{gathered}
M\left(N, q \cdot p^{k}\right) \supset M^{a}\left(\cdot, q \cdot p^{k-1}\right) \supset \cdots \\
\quad \supset M^{a}(\cdot, q \cdot p) \supset M^{a}(\cdot, q)
\end{gathered}
$$

which is accompanied by an ordering analogous to (11).

The last manifold $M^{a}(N, q)$ in (12) has a continuation of submanifolds depending on the number $q$.

Rule 4 In the general case $N=p_{1}^{k_{1}}, p_{2}^{k_{2}}, \ldots, p_{e}^{k_{e}}$, where all $p_{j}$ are prime numbers, for each cofactor $p_{j}^{k_{j}}$ placed to the end ( $n=p_{j}^{k_{j}}$ in Theorem 2) the branch of embedded manifolds is generated in accordance with Rule 3. Each pair of such branches is disjoint for higher dimensions and may be merged at low dimensions of embedded manifolds.

In what cases will the array of coupled maps (2) have 2 clusters? According to our considerations 
the answer is the following, only

(1) if $N$ is even, $N=N^{\prime} \cdot 2$, and system (2), therefore due to Theorem 2 has the manifold $M^{a}(N, 2)$ with the map

$$
\begin{aligned}
& \bar{x}_{1}=F\left(x_{1}\right)+\varepsilon \cdot G\left(x_{1}, x_{1}, x_{2}\right), \\
& \bar{x}_{2}=F\left(x_{2}\right)+\varepsilon \cdot G\left(x_{1}, x_{2}, x_{2}\right)
\end{aligned}
$$

on it, or

(2) if number 3 is cofactor of $N, N=N^{\prime \prime} \cdot 3$, and array (2) therefore due to Theorem 2 has the manifold $M^{a}(N, 3)$ with the map

$$
\begin{aligned}
& \bar{x}_{1}=F\left(x_{1}\right)+\varepsilon \cdot G\left(x_{1}, x_{1}, x_{2}\right), \\
& \bar{x}_{2}=F\left(x_{2}\right)+\varepsilon \cdot G\left(x_{1}, x_{2}, x_{3}\right), \\
& \bar{x}_{3}=F\left(x_{3}\right)+\varepsilon \cdot G\left(x_{2}, x_{3}, x_{3}\right)
\end{aligned}
$$

on it.

In turn, due to Theorem 1 , the manifold $M^{a}(N, 3)$ has the submanifold $M^{c}(3,2)$ with the map

$$
\begin{aligned}
& \bar{x}_{1}=F\left(x_{1}\right)+\varepsilon \cdot G\left(x_{1}, x_{1}, x_{2}\right), \\
& \bar{x}_{2}=F\left(x_{2}\right)+\varepsilon \cdot G\left(x_{1}, x_{2}, x_{1}\right)
\end{aligned}
$$

on it.

Note that the map (13) due to (3) has the symmetry $x_{1} \Leftrightarrow x_{2}$. This situation was considered, for instance, in [4].

As examples we list some cases of interesting numbers of individual subsystems (1) composing the array (2).

Example 1 Let the number of array elements $N=15$. In this case there exists the following ordering of embedded manifolds:

$$
\begin{gathered}
\supset M(15,3) \supset \\
M(15,15) \supset M(15,8) \supset \quad \supset(15,2) \\
\qquad M(15,1) . \\
\supset M(15,5) \supset
\end{gathered}
$$

Example 2 Let $N=12$. Then there exists an ordering of embedded manifolds

$$
\begin{aligned}
& \supset M(12,4) \\
& M(12,12) \supset M(12,2) \supset \\
& \supset M(12,6) \supset M(12,3) \supset \\
& \supset \quad \supset M(12,1) . \\
& \supset M^{\prime}(12,2)
\end{aligned}
$$

\section{TRANSVERSAL INVARIANT MANIFOLDS}

Let us consider the system (2) under the additional conditions on the functions in (2) to be odd:

$$
\begin{aligned}
F(x) & =-F(x), \\
G(u, v, w) & =-G(-u,-v,-w) .
\end{aligned}
$$

In this case the system (2) is centrally symmetric with respect to the zero point $x=0$ which is a fixed point of the map $T$.

THEOREM 4 Under conditions (4) and (16) with zero-flux boundary conditions, the map (2) has an $n$-dimensional invariant manifold $M^{\mathrm{T}}(N, n)$ with $\operatorname{int}((N+1) / 2)$ written in the form

$$
M^{\mathrm{T}}(N, n)=\left\{x_{i}=-x_{N-i+1}, i=1,2, \ldots, n\right\}
$$

for even $N=2 n$, and in the form

$$
\begin{aligned}
M^{\mathrm{T}}(N, n)= & \left\{x_{i}=-x_{N-i+1}, i=1,2, \ldots, n,\right. \\
& \left.x_{n+1} \equiv 0\right\}
\end{aligned}
$$

for odd $N=2 n+1$. The correspondence matrix has the form $C=\operatorname{column}(E,-\bar{E})$ for even $N$ and $C=\operatorname{column}(E, 0,-\bar{E})$ for odd $N$.

Proof The proof of the theorem follows from the fact that the last $n$ equations of (2) after the substitution $x_{N-i+1}=x_{i}$ reduce to the first $n$ equations with the opposite sign of each term. For odd $N$ the function

$$
\left.G\left(x_{n}, x_{n+1}, x_{n+2}\right)\right|_{M^{\mathrm{T}}}=G\left(x_{n}, 0,-x_{n}\right)=0
$$


and

$$
\left.F\left(x_{n+1}\right)\right|_{M^{\mathrm{T}}}=F(0) \equiv 0 .
$$

Hence $\left.\bar{x}_{n+1}\right|_{M^{\mathrm{T}}} \equiv 0$.

COROLlary Theorem 4 can be applied to each ordinary cluster in $M(N, n)$ deriving from Theorems 1 and 2 and having zero-flux boundary conditions. Hence, we obtain similar rules of generation of the families of manifolds. For example, in the case $N=5$ we obtain the manifold $M^{\mathrm{T}}(5,2)$ as well as for $N=15$ besides the manifold $M^{\mathrm{T}}(15,7)$, the manifold $M(15,5)$ has a transversal submanifold $M^{\mathrm{T}}(15,2)$.

Remark 1 In the case of odd $N$ the manifold $M^{\mathrm{T}}(N, n)$ is the invariant submanifold of the zero fixed point.

Remark 2 Similarly to the case of continuous time dynamical systems coupled in an array, the orbits in the transversal manifolds may be related to anti-phase synchronous orbits.

The orderings described in Sections 3-5 determine the existence of all permitted manifolds and therefore all possible cluster structures defined by stable linear invariant manifolds. They may also determine the order of cluster regime appearance with changing coupling between the individual subsystems of the array (2). Thus after having considered the conditions for the existence of invariant manifolds, the main problem is to obtain the conditions for their stability. That is the topic of Section 5 .

\section{STABILITY OF INVARIANT MANIFOLDS}

The Lyapunov local stability of an invariant manifold $M(N, n)$ implies that this particular manifold is "observable" and hence that it corresponds to partial synchronization of $N$ elements distributed in $n$ synchronous clusters. Hereafter we present the variational linear equations for the simple case of system (2). We consider the one-dimensional single map

$$
\bar{x}=f(x), \quad f: R^{1} \rightarrow R^{1}
$$

and the CML with nonlinear coupling defined by the scalar function $g(x), g: R^{1} \rightarrow R^{1}$, i.e., we consider the map (1) with zero-flux boundary conditions (4).

Equation (1) may be written in the vector form. Introducing the vectors

$$
\begin{aligned}
X & =\left(x_{1}, x_{2}, \ldots, x_{N}\right)^{\mathrm{T}}, \\
\Phi(X) & =\left(f\left(x_{1}\right), f\left(x_{2}\right), \ldots, f\left(x_{N}\right)\right)^{\mathrm{T}}, \\
Q(X) & =\left(g\left(x_{1}\right), g\left(x_{2}\right), \ldots, g\left(x_{N}\right)\right)^{\mathrm{T}}
\end{aligned}
$$

and the coupling matrix

$$
S=\left(\begin{array}{ccccc}
-\varepsilon & \varepsilon & 0 & \cdots & 0 \\
\varepsilon & -2 \varepsilon & \varepsilon & 0 & \vdots \\
0 & \varepsilon & \ddots & \ddots & 0 \\
\vdots & 0 & \ddots & -2 \varepsilon & \varepsilon \\
0 & \cdots & 0 & \varepsilon & -\varepsilon
\end{array}\right),
$$

system (1) attains the form

$$
\bar{X}=\Phi(X)+S \cdot Q(X) .
$$

As before we denote the vector of cluster coordinates $U=\left(x_{1}, x_{2}, \ldots, x_{n}\right)^{\mathrm{T}}$.

Let $C$ be the matrix from Theorems 1 and 2 (picking out the coordinates of $X$ into a particular cluster). Let us study the conditions of stability of the manifold $M(N, n)$.

Consider the variance vector $Y=C U-X$ and its image $\bar{Y}=C \bar{U}-\Phi(X)-S Q(X)$. Since the upper part of $C$ is the identity matrix, the first $n$ coordinates of $Y$ and $\bar{Y}$ are zeros. Preserving the notations for $Y$ and $C$ having removed the first $n$ lines, for small $Y$ we obtain the variational linear equation

$$
\bar{Y}=\left[\Phi_{x}(C U)+S Q_{x}(C U)\right] \cdot Y \equiv L(u) Y,
$$

where $\Phi_{x}$ and $C_{x}$ denote the vectors of derivatives of $f$ and $g$, respectively. $Y$ represents the difference between coordinates $\left(x_{n+1}, x_{n+2}, \ldots, x_{N}\right)$ and cluster coordinates distributed by the correspondence matrix $C$. The $n$-vector $U$ in (18) is driven by the 
map in the manifold $M(N, n)$

$$
\bar{U}=\Phi(U)+\tilde{S} Q(U)
$$

where $\tilde{S}$ is the matrix $S$ with $N$ replaced by $n$ and the last line in the matrix changed in the case of asymmetrical boundary conditions.

Thus, the system of Eqs. (18) and (19) allows us to calculate the Lyapunov exponents for each trajectory from the limiting set filling out the attractors of the system (19) and hence to characterize the local stability of the manifold $M(N, n)$. In the general case of chaotic maps (19) it is hard to avoid computation of the eigenvalues of the products of matrices $L(u)$ along trajectories of the map (19). Nevertheless, it is possible to estimate the multipliers for certain cases of $f(x)$ and $g(x)$ in (1).

Example Let $N=3$ and assume that we have an array of 3 locally coupled one-dimensional maps. Consider the two-dimensional manifold $M(3,2)=$ $\left\{x_{1}=x_{3}\right\}$. In this case

$$
C=\left(\begin{array}{ll}
1 & 0 \\
0 & 1 \\
1 & 0
\end{array}\right)
$$

and

$$
Y=C U-X=\left(\begin{array}{l}
x_{1} \\
x_{2} \\
x_{1}
\end{array}\right)-\left(\begin{array}{l}
x_{1} \\
x_{2} \\
x_{3}
\end{array}\right)=\left(\begin{array}{c}
0 \\
0 \\
x_{1}-x_{2}
\end{array}\right) .
$$

Then for (18) and (19), respectively we obtain

$$
\begin{aligned}
& \bar{y}_{1}=\left[f^{\prime}\left(x_{1}\right)-\varepsilon \cdot g^{\prime}\left(x_{1}\right)\right] y_{1}, \\
& \bar{x}_{1}=f\left(x_{1}\right)+\varepsilon\left(g\left(x_{2}\right)-g\left(x_{1}\right)\right), \\
& \bar{x}_{2}=f\left(x_{2}\right)+2 \varepsilon\left(g\left(x_{1}\right)-g\left(x_{2}\right)\right) .
\end{aligned}
$$

For the diagonal one-dimensional manifold $M(3,1)=\left\{x_{1}=x_{2}=x_{3}\right\}$ we obtain for $y_{1}=x_{1}-x_{3}$, $y_{2}=x_{1}-x_{2}$ the following variational equations:

$$
\begin{aligned}
& \bar{y}_{1}=\left[f^{\prime}\left(x_{1}\right)-\varepsilon \cdot g^{\prime}\left(x_{1}\right)\right] y_{1}, \\
& \bar{y}_{2}=\left[f^{\prime}\left(x_{1}\right)-3 \varepsilon \cdot g^{\prime}\left(x_{1}\right)\right] y_{2}+\varepsilon \cdot g^{\prime}\left(x_{1}\right) y_{1},
\end{aligned}
$$

where $x_{1}$ is driven by the single map $\bar{x}_{1}=f\left(x_{1}\right)$. The embedding $M(3,1) \subset M(3,2)$ implies that for $x_{1}=x_{2}$ in (21) Eq. (20) coincides with the first equation in (22) both being driven by a single map. On the other hand, the stability of the diagonal $x_{1}=x_{2}$ submanifold for (21) is determined by the second equation in (22) for $y_{1}=0$.

Note that even for this simple case we meet the problem of how to choose the coupling function $g(x)$ in order to provide stability of the twodimensional manifold for some range of parameter $\varepsilon$ and inside of this range to provide stability of the manifold $M(3,1)$ for a smaller interval of $\varepsilon$. We need this stability since only in this case may partial synchronization be realized (the manifold $M(3,2)$ must be stable and the diagonal $M(3,1)$ must be unstable). In the alternative case when the manifolds $M(3,1)$ and $M(3,2)$ acquire their stability exactly in the same region of parameter $\varepsilon$, only the dynamical regime of full synchronization exists.

We present two simple examples for sufficient conditions of stability of (1) for $N=3$.

Case 1 Let $g(x)=f(x)$, and the map (1) becomes a Kaneko-type map. Assume that both the single map $\bar{x}=f(x)$ and the map (21) are chaotic and have the attractors $A_{1}$ and $A_{2}$ as limiting sets, respectively.

If the conditions

$$
\begin{aligned}
& \max _{x \subset A_{1}}|1-\varepsilon| \cdot\left|f^{\prime}(x)\right|<1, \\
& \max _{x \subset A_{1}}|1-3 \varepsilon| \cdot\left|f^{\prime}(x)\right|<1
\end{aligned}
$$

hold, then the manifold $M(3,1)$ is globally asymptotically stable and full synchronization of the individual subsystems occurs.

If the condition

$$
\max _{x \subset A_{2}}|1-\varepsilon| \cdot\left|f^{\prime}(x)\right|<1
$$

holds, then the manifold $M(3,2)$ is globally asymptotically stable and partial synchronization (synchronization between the first and the third element) arises. 
Note that if for both cases $\max _{x \subset A_{1,2}}\left|f^{\prime}(x)\right|=$ $1+\alpha>1$, where $\alpha$ is some positive parameter needed for the single system be chaotic. Then conditions (23) on the coupling parameter $\varepsilon$ take a form

$$
\frac{\alpha}{\alpha+1}<\varepsilon<\frac{\alpha+2}{3(\alpha+1)}, \quad 0<\alpha<1
$$

and instead of condition (24) we have

$$
\frac{\alpha}{\alpha+1}<\varepsilon<\frac{\alpha+2}{(\alpha+1)} \text {. }
$$

From (25) and (26) it follows that if for $\varepsilon>(\alpha+2) /$ $(3(\alpha+1))$ the minimal multiplier of the trajectories in $A_{2}$ is greater than 1. Then chaotic partial synchronization takes place, while full synchronization is absent.

Case 2 Let $g(x)=x$ the map (1) has linear diffusive coupling, and $N=3$. Again assuming that both the single map and the map (21) have chaotic attractors $\tilde{A}_{1}$ and $\tilde{A}_{2}$, respectively, we obtain the following sufficient conditions for full synchronization

$$
\begin{aligned}
& \max _{x \subset A_{1}}\left|f^{\prime}(x)-\varepsilon\right|<1, \\
& \max _{x \subset A_{1}}\left|f^{\prime}(x)-3 \varepsilon\right|<1
\end{aligned}
$$

and of partial synchronization

$$
\max _{x \subset A_{2}}\left|f^{\prime}(x)-\varepsilon\right|<1 .
$$

For the chaotic single map, the function $f(x)$ must have both positive and negative slopes. In this case the sufficient conditions (27) and (28) seem to be incompatible, and in order to solve the problem of synchronization we need the detailed analysis of both trajectories of attractors $\tilde{A}_{1}$ and $\tilde{A}_{2}$ and their Lyapunov exponents via systems (20)-(22) for $g(x)=x$. In the case of three diffusively coupled one-dimensional discontinuously expanding maps having

$$
f(x)=(1+\beta) x(\bmod 1)
$$

we obtain the following condition on stability of full synchronization:

$$
\beta<\varepsilon<\frac{\beta+2}{3}, \quad \beta<1
$$

and of partial synchronization

$$
\begin{gathered}
\frac{\beta+2}{3}<\varepsilon<\beta+2, \quad \beta<1 ; \\
\beta<\varepsilon<\beta+2, \quad \beta>1 .
\end{gathered}
$$

Remark 1 For the purpose of estimation of global stability of the manifold for large values of $\|Y\|$ it is possible to consider Eq. (18) with the vector $U$ replaced by a vector $\bar{U}(u)$ coming from the Lagrange mean-value theorem.

Remark 2 The equations for stability of the general system (2) can be derived in the similar way where each element of the matrix $L(u)$ becomes an $m \times m$ matrix.

Finally, we discuss the stability of invariant manifolds in the case of a number of individual elements $N=2^{k}$. The system (2) has the properties of self-similarity of the manifolds $M^{c}\left(2^{k}, 2^{k-1}\right)$, $\ldots, M^{c}\left(2^{k}, 2^{k-j}\right), \ldots, M^{c}\left(2^{k}, 2\right)$ implying that the map $\left.T\right|_{M^{c}\left(2^{k}, 2^{k-j}\right)}$ is the same as the original map, but with a phase space reduction by the factor $2^{j}$. For rather small coupling strength $\varepsilon$, all elements of the array are desynchronized. The changes in system dynamics while parameter $\varepsilon$ increases may occur in the following way. First the diagonal $M^{c}\left(2^{k}, 1\right)$ becomes stable relating to the trajectories inside of the manifold $M^{c}\left(2^{k}, 2\right)$, i.e., for the map $\left.T\right|_{M^{c}\left(2^{k}, 2\right)}$ though the manifold $M^{c}\left(2^{k}, 2\right)$ is unstable with respect to the manifold $M^{c}\left(2^{k}, 4\right)$, i.e. for the map $\left.T\right|_{M^{c}\left(2^{k}, 2\right) \text {. Then the pairs in array elements }}$ (already "prepared" for full synchronization) obtain additional stability with respect to the manifold $M^{c}\left(2^{k}, 4\right)$ and so forth. At the last step, when the manifold $M^{c}\left(2^{k}, 2^{k-1}\right)$ becomes stable (now with respect to phase space $M^{c}\left(2^{k}, 2^{k}\right)$ ), full synchronization arises in a hard way. So in this stabilization process of invariant manifolds, partial synchronization is not observed for any coupling parameter value and it is relevant to use the term 
conditional partial synchronization for the stability of the manifold $M^{c}\left(2^{k}, 2^{k-j}\right)$ with respect to the map $\left.T\right|_{M^{c}\left(2^{k}, 2^{k-j+1}\right)}$.

This gradual increase of stability of invariant manifolds is a particular possible scenario of bifurcations leading to full synchronization. In the general case this ordering may be broken at any place. In this connection the problem arises to find a map $F$ and a coupling function $G$ in (2) providing the reverse ordering of stability for $N=2^{k}$ (or $\left.N=p^{k}\right)$ such that manifold $M^{c}\left(2^{k}, 2 k-j+1\right)$ becomes stable before the manifold $M^{c}\left(2^{k}, 2^{k-j}\right)$ does. This particular problem is within the general problem of stability of any manifold $M(N, n)$ under the condition of simultaneous instability of
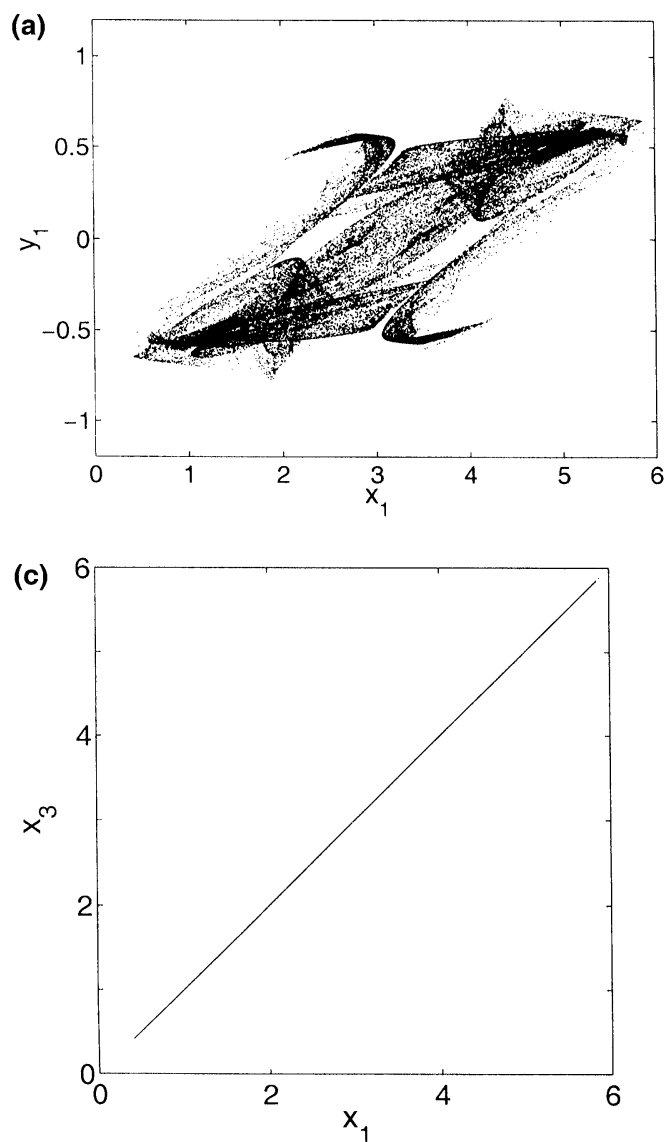

the diagonal $M(N, 1)$, i.e. the problem of partial (cluster) synchronization realization.

In the next section we exhibit this possibility for example of coupled standard maps with damping.

\section{EXAMPLE}

Consider as a computational example of three coupled two-dimensional standard maps with damping $[5,12]$ :

$$
\begin{aligned}
& \bar{x}_{i}=x_{i}+y_{i}+a \sin x_{i}+\varepsilon_{1} \cdot\left(x_{i-1}-2 x_{i}+x_{i+1}\right) \\
& \bar{y}_{i}=\lambda\left(y_{i}+a \sin x_{i}\right)+\varepsilon_{2} \cdot\left(x_{i-1}-2 x_{i}+x_{i+1}\right),
\end{aligned}
$$
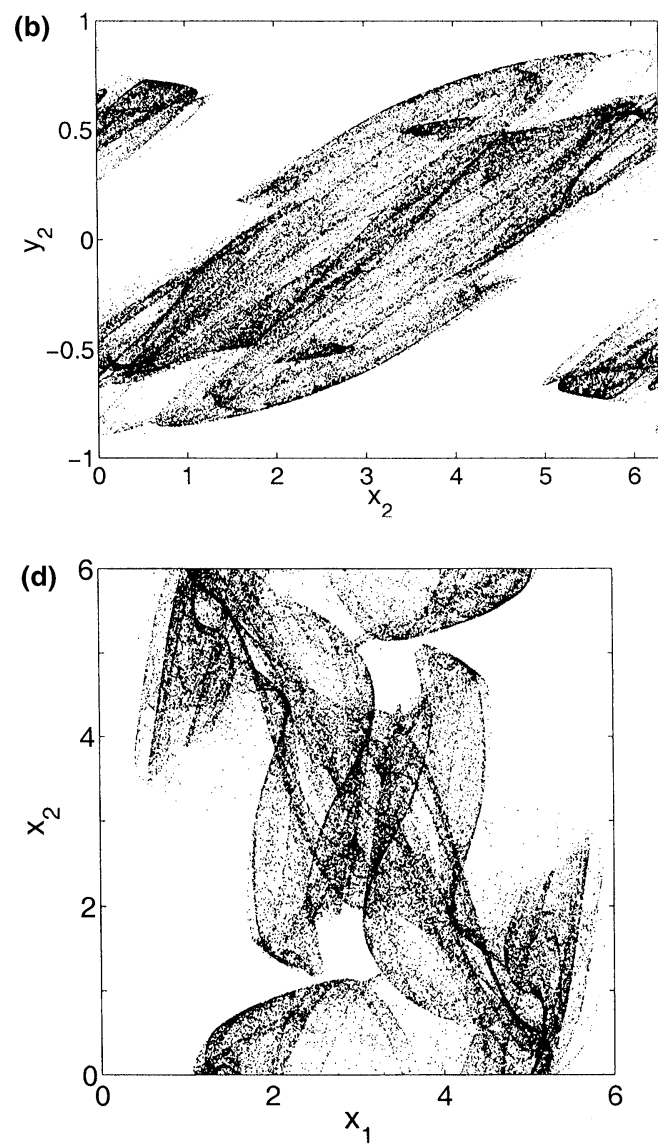

FIGURE 1 Chaotic cluster synchronization of three coupled damped standard maps. Case $\varepsilon_{1} \neq 0, \varepsilon_{2}=0$. (a) Lying in the stable manifold $M(3,2)$ we observe the chaotic attractor in a projection onto $\left(x_{1}, y_{1}\right)$. (b) Chaotic attractor in the projection of variables of the middle element $\left(x_{2}, y_{2}\right)$. (c) Stable manifold $M(3,2)$ defining the synchronization between the first and the last element of the array. (d) Out of synchronization between the first (the last) and the middle element. 
(a)

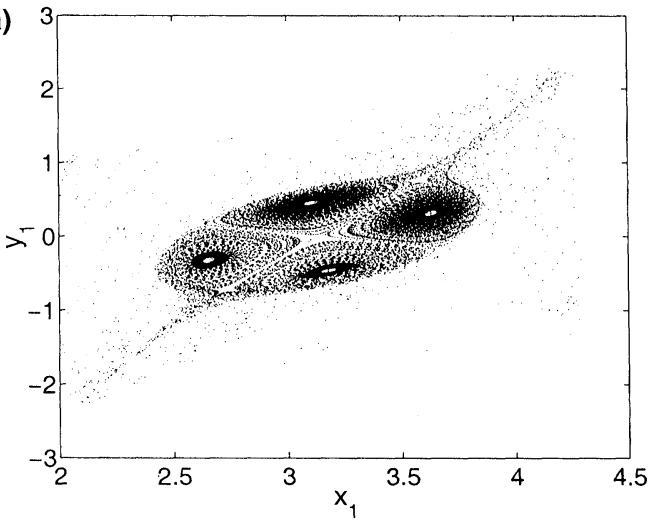

(c)

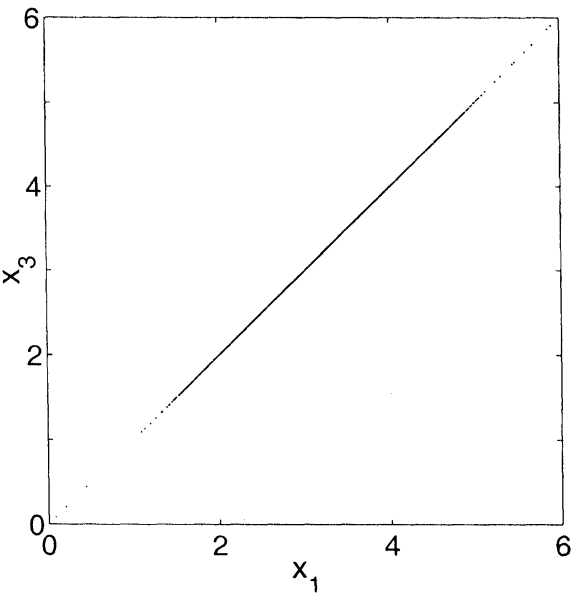

(b)

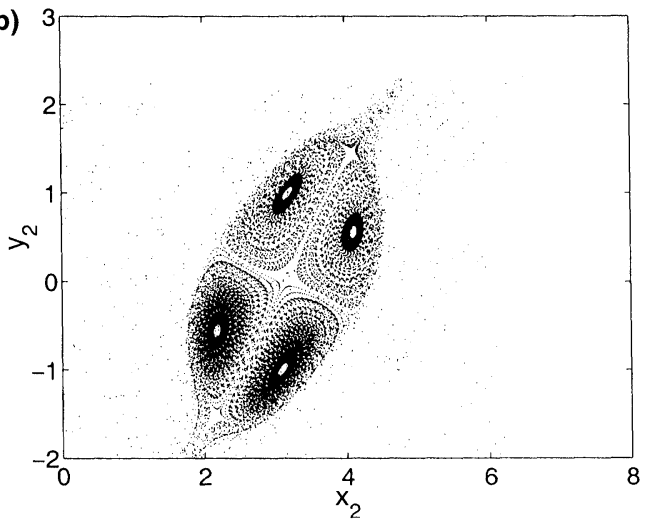

(d)

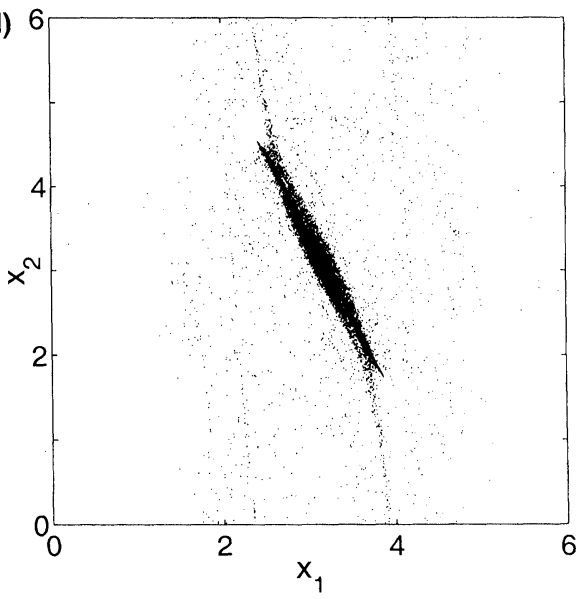

FIGURE 2 Chaotic cluster synchronization of three coupled damped standard maps. Case $\varepsilon_{1}=0, \varepsilon_{2} \neq 0$. (a) Chaotic attractor in the projection on variables of the first map $\left(x_{1}, y_{1}\right)$. (b) Chaotic attractor in the projection of variables of the middle element $\left(x_{2}, y_{2}\right)$. (c) Stable manifold $M(3,2)$ which determines cluster chaotic synchronization. (d) Out of full synchronization.

$\bar{x}_{i}(\bmod 2 \pi), \quad i=1,2,3$ with zero-flux boundary conditions (3). This map has the manifold $M(3,2)=$ $\left\{x_{1}=x_{3}, y_{1}=y_{3}\right\}$ as well as the full synchronization manifold $M(3,1)=M(3,2) \mid\left\{x_{1}=x_{2}, y_{1}=y_{2}\right\}$. We have chosen the coupling matrix

$$
E_{p}=\left(\begin{array}{ll}
\varepsilon_{1} & 0 \\
\varepsilon_{2} & 0
\end{array}\right)
$$

thus excluding the terms with combination $\left(y_{i-1}-2 y_{i}+y_{i+1}\right)$, in order to exhibit the difference in dynamical behavior in the two cases:

(1) $\left(\varepsilon_{1} \neq 0, \varepsilon_{2}=0\right)$ when the coupling term is placed in the first equation of the two-dimensional standard map, and Eq. (29) may be reduced for $\lambda=0$ to three coupled one-dimensional circular maps;

(2) $\left(\varepsilon_{1}=0, \varepsilon_{2} \neq 0\right)$ when the coupling is placed in the second equation, i.e. in the proper place if the single map is treated as a two-dimensional discrete oscillator.

Figure 1 illustrates chaotic partial synchronization in the first case ( parameters are $a=2.4, \lambda=0.3$, $\varepsilon=0.45)$. Note, that the unusual behaviors of the trajectories in both clusters $\left(x_{1}, y_{1}\right)=\left(x_{3}, y_{3}\right)$ and $\left(x_{2}, y_{2}\right)$ are related to the imposed periodicity via $\bar{x}_{i}(\bmod 2 \pi)$.

Figure 2 illustrates chaotic partial synchronization in the second case (parameters are $a=1.8$, 
$\lambda=0.6, \varepsilon=-0.89)$. The behavior of the clusters confirm the oscillatory nature of the equations.

We omit the discussions of nontrivial bifurcation sets of (29), using (29) as a particular example of the existence of a stable invariant manifold with chaotic dynamics.

\section{Acknowledgments}

This work was supported in part by RFFI (grant No. 99-01-01126) and by grant "Universities of Russia" (No. 1905). V.B. and I.B. acknowledge support from the Danish Research Academy.

\section{References}

[1] K. Kaneko Physica D 23, 436 (1986); 41, 137 (1990); 54, 5 (1991); 55, 368 (1992); 75, 55 (1994).
[2] V.S. Anishenko, I.S. Aranson, D.E. Postnov and M.I. Rabinovich Doklady Akademii Nauk, SSSR, 286(5), 1120 (1986).

[3] V.S. Afraimovich and V.I. Nekorkin Int. J. Bif. Chaos 4, 631 (1994).

[4] C. Reick and E. Mosekilde Phys. Rev. E 52, 1418 (1995).

[5] V.N. Belykh and E. Mosekilde Phys. Rev. E, 54(4), 3196 (1996).

[6] A.S. Dmitriev, M. Shirokov and S.O. Starkov IEEE Trans. on Circuits and Syst. 44(10), 918 (1997).

[7] L.M. Pecora, T.L. Carroll, G.A. Johnson, D.J. Mar and J.F. Heagy Chaos 7(4), 520 (1997).

[8] M. Hasler, Yu. Maistrenko and O. Popovich Phys. Rev. E, 58(5), 6843 (1999).

[9] Yu.L. Maistrenko, V.L. Maistrenko, A. Popovich and E. Mosekilde Phys. Rev. E 60(3), 2817 (1999).

[10] V.N. Belykh Int. Conf. Diff Eq., Moscow Aviation Institute, Steklov Inst. Math. RAS, Moscow, 13 (1999).

[11] I.V. Belykh Ph.D. Theses, Nizhny Novgorod University (1999).

[12] V.N. Belykh Math. Russ. Sbornik 186(3) (1995).

[13] V.N. Belykh, I.V. Belykh and M. Hasler Phys. Rev E (to appear). 


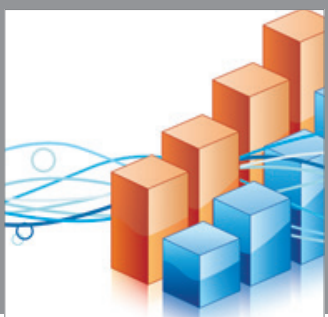

Advances in

Operations Research

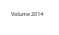

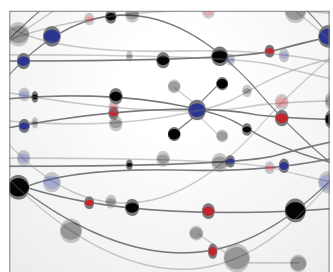

\section{The Scientific} World Journal
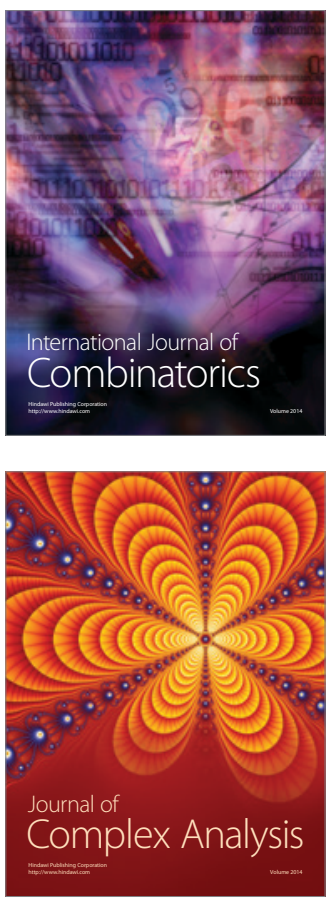

International Journal of

Mathematics and

Mathematical

Sciences
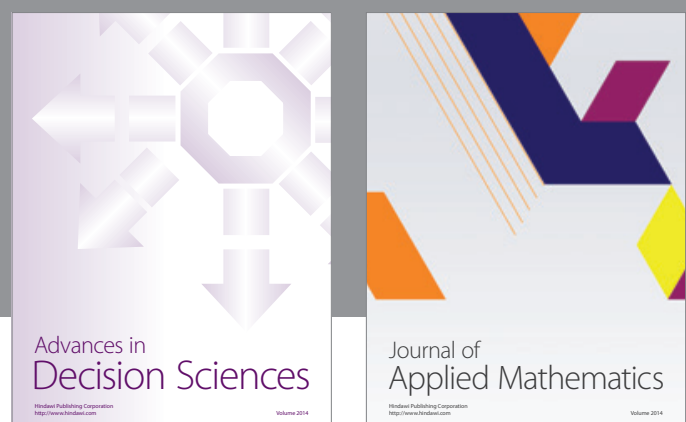

Journal of

Applied Mathematics
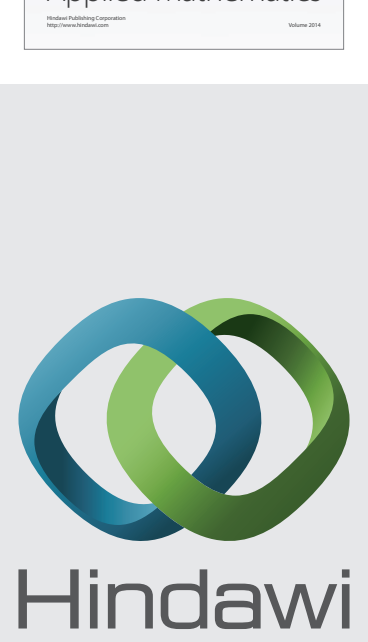

Submit your manuscripts at http://www.hindawi.com
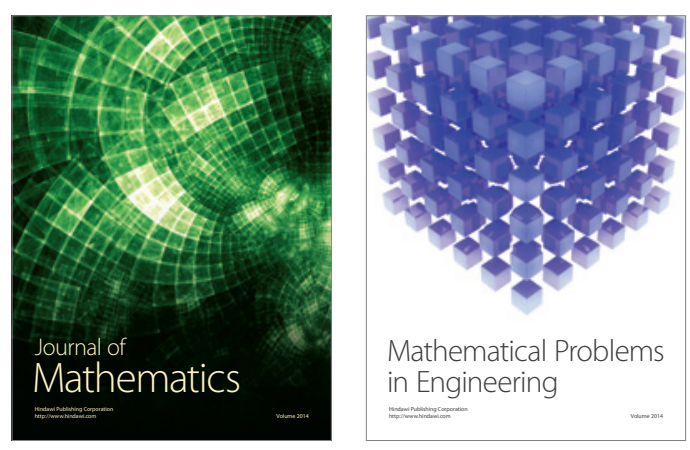

Mathematical Problems in Engineering
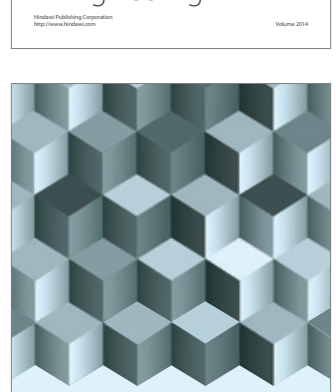

Journal of

Function Spaces
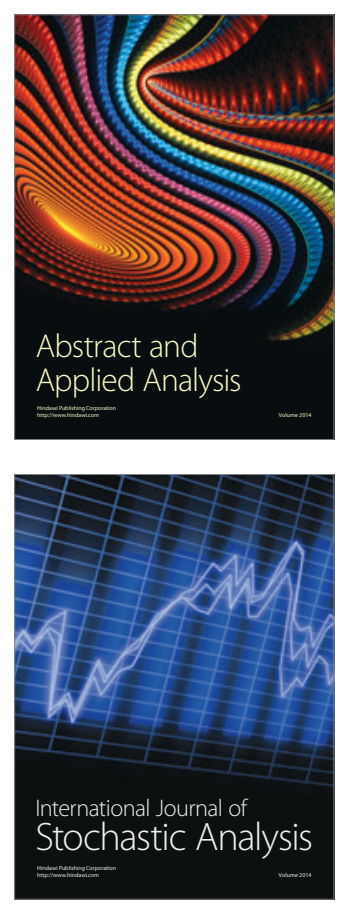

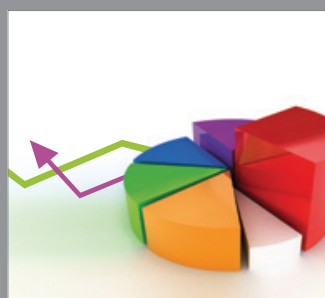

ournal of

Probability and Statistics

Promensencen
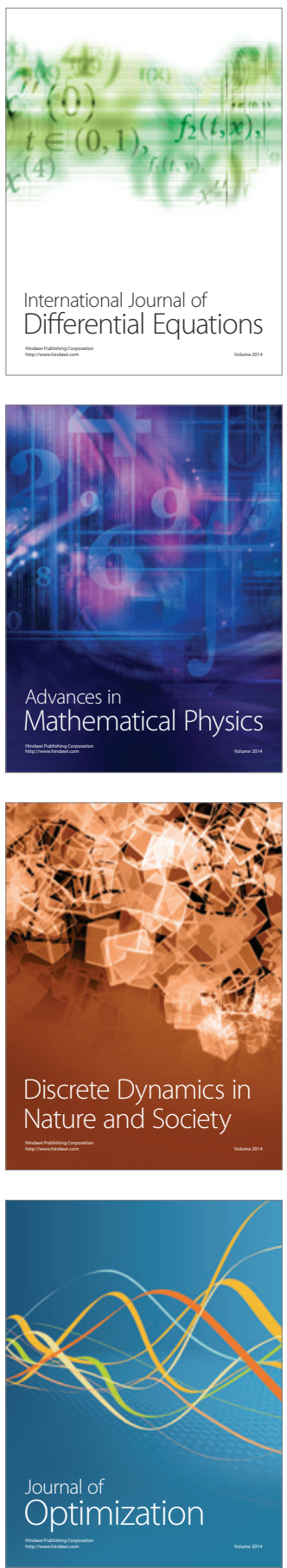